\title{
BREAST CANCER DETECTION USING ANN NETWORK AND PERFORMANCE ANALYSIS WITH SVM
}

\author{
Kalyani Wadkar \\ Department of Computer Science and Engineering \\ Government College of Engineering, Aurangabad, Maharashtra, India \\ Prashant Pathak \\ Department of Computer Science and Engineering \\ Government College of Engineering, Aurangabad, Maharashtra, India \\ Nikhil Wagh \\ Department of Computer Science and Engineering \\ Government College of Engineering, Aurangabad, Maharashtra, India
}

\begin{abstract}
According to the World Health Organization (WHO) breast cancer is the major reason of death among women and its impact on women is 2.1 million per year. Only in 2018 approximately 15\% (62700) of women are died due to breast cancer. To detect this breast cancer oncologist rely on two methods i.e. early diagnosis and screening. To identify cancers before any symptoms appear screening plays an important role and in screening Mammography is heart of breast cancer detection. Apart from this Clinical Breast Exams, Breast Self-Exam and many other methodologies are used. Screening for breast cancer is too long and time consuming process if approach is manual analysis and it's performed on medical images. It's also unfeasible for huge data sets. That's the reason we required self-automated, efficient and more accurate machine to identify or capture the breast cancer as minimum as possible amount of time. We found the solution of this problem is Deep Learning Method. It provides the results in short period of time as compare to other techniques and giving the better accuracy for detection of Breast cancer. In this paper we focuses on, by using which methodology we got the more accurate results and how much amount of time is required to do this process. In this project we are going to deal with different classifiers like CNN, KNN, Inception V3, $S V M$ and ANN. By using ANN we are going to detect the Breast Cancer. We are also going to compare the results of SVM with ANN Technique.
\end{abstract}

Keywords: Breast Cancer (BC), Deep Learning, Artificial Neural Network (ANN), Support Vector Machine (SVM), K-Nearest neighbor (KNN), Convolutional Neural Network (CNN). 
Cite this Article: Kalyani Wadkar, Prashant Pathak and Nikhil Wagh, Breast Cancer Detection using Ann Network and Performance Analysis with SVM, International Journal of Computer Engineering and Technology, 10(3), 2019, pp. 75-86.

http://iaeme.com/Home/issue/IJCET?Volume=10\&Issue=3

\section{INTRODUCTION}

One of the most harmful disease in the world is cancer and the cancer which killed the women most is Breast Cancer (BC) [1]. One of the study says it frequently occur in women those age is greater than 40 and on of the reason is it takes large amount of time for detecting the Breast Cancer (BC) manually [2] [1]. That's the reason we required automated diagnostic tools or techniques to detect and classify the $\mathrm{BC}$ in minimum amount of time. There are some methods which already working on detection of BC i.e. Mammogram, Ultrasound method then Magnetic Resonance Imaging and biopsy.

Doctors says that BC happened due to abnormal growth of cells in breast and this cells spread in size like MetaSize from breast to lumph nodes or to the other parts of body also [3]. Hence it is necessary to detect and stop the growth of this unwanted cells as early as possible to avoid the next phase consequences [1]. If tumor is diagnosed then the first step taken by doctor is they checks whether the tumor is Benign or Malignant. Because the treatment and prevention methods of both the tumors are different. In Benign cells are neither cancerous and nor they spreads but in Malignant cells are cancerous and can spread to other parts of bodies [4] [1]. The problem with this disease is, there is no such proper diagnostic machine is present to detect the cancer in early phase so the person can start the treatment as early as possible and try to stop the growth of unwanted cells or tumor [1]. In today's technological world if we want to achieve the better results than we have to move towards computerized diagnostic system in which machine learning methodology is used. This methodology includes algorithms which helps for classification of tumor and detect the cells more accurately and take less time as well [1].

This work is based on the predicting the BC by using deep learning method called Artificial Neural Network and compare the results with Support Vector Machine.

\section{LITERATURE SURVEY}

Many researchers study the machine learning methodology and compare many classification techniques or algorithms and analyze the results.

|Lal Hussan, et-al, 2018| this work is based on distinguishes the cancer from normal images by using various Machine Learning Classification algorithms like Decision Tree, Bayesian approach and SVM kernel. AUC, NPV, FPR, sensitivity and specificity are used to measure the performance. The results shown that the different features extracting methods and classifiers gives us highest performance for detection of BC Mammograms [11].

|Siyabend Turgut, et-al, 2018| works on finding the performance of SVM after the feature selection technique and it gives better results than the normal one. The accuracy of the dataset is increase after the feature selection method [5].

|Liu Lei, 2018| works on classification techniques on the basis of two characteristics mean radius and mean texture. This module got the accuracy up to $90.48 \%$ and with the characteristics maximum texture and maximum parameter accuracy is up to $96.5 \%$. The second combination gives the better results as compare to first combination and we called it as Logistic Regression. It gives results in less time and more efficiently [10].

|Dr. D. Selvathi, et-al, 2017| works on classification techniques and they cover SVM, KNN, Random Forest and Decision Tree techniques for detecting BC with the help of Mammographic 
images. They first prepared the image and remove noises for better results. Radio-opaque artifacts, background and pictorial muscle and their model achieved an accuracy is $98.9 \%$ for unsupervised learning [2].

|Phonethep Doungnoulack, et-al, 2017| works on three classifiers J48, REP Tree and Random Tree. They performed the operation on the data with PCA and without PCA reduction. They got the results for detecting $\mathrm{BC}$ is, $\mathrm{J} 48$ classifier gives better accuracy as compare to other and that is $97.36 \%$ (REP Tree-96.77\% and Random Tree-97.36\%) [6].

|Md. Milon Islam, et-al, 2017| works on prediction of BC using SVM and KNN. SVM implemented by using python which is effective method for classification of diagnostic data into two different classes. This model gives the accuracy for SVM is $99.68 \%$ in training phase. Results are obtained by supervised learning [7].

|S. Murugan, et-al, 2017| works on three algorithm Random Forest, Liner regression and Decision Tree for prediction of $\mathrm{BC}$ and the success rate of liner regression classification is $84.14 \%$ and for random forest is $88.14 \%$ [8].

|Ahmed Iqbal Pritom, et-al, 2016| works on finding better BC prediction method i.e. SVM after attribute selection Decision Tree and Naïve Bayes techniques which gives better performance after feature selection. They focuses on finding BC is a recursive or not [9].

\section{PROPOSED WORK ARCHITECTURE}

The flow of proposed model as shown in figure 1. It shows that how the data set is processed step by step in system and giving the results.

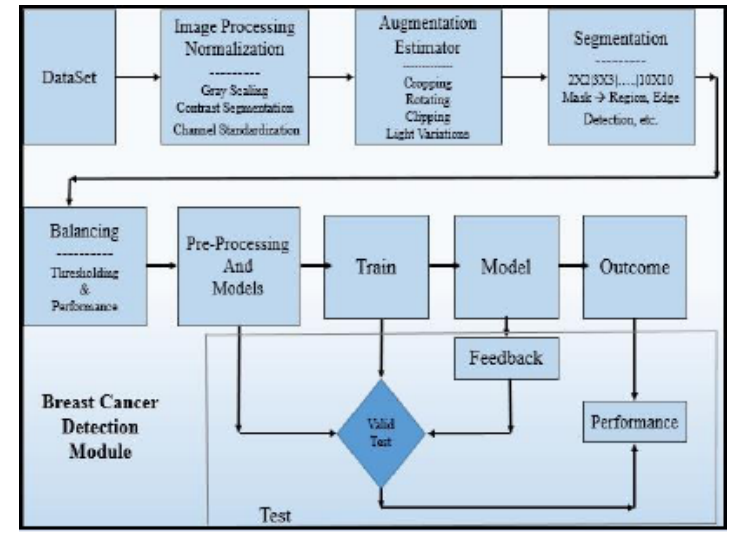

Figure 1 Proposed Architecture for BC detection phase.

The steps shown in above figure is explain below one by one. Let's see the working of each

\subsection{Data Set}

We are taking data by considering supervised learning methodology. We first train the machine with sorted data which contains the type like Benign and Malignant and split that data with different attribute like training, validation and Test sets. Here we write one script for automatic selection of images from the location we provided in scaled numpy matrix and every single image sample in 0 or 1 format. To train machine bitterly we split and arrange data in proper format for giving more accuracy in test phase [12] [13].

\subsection{Image Processing Normalization}

We know that the dataset contain large number of images and all this images contain different level of blurriness or staining. This is vary from lab to lab, what method or what protocol they 
are using for collection of the tissue. To train and process the data properly we select the normalization method for color validation. It gives us better accuracy in letter phase. We followed three different approaches in normalization [12].

- Gray Scaling: Its convert normal images into grayscale format somewhat like black and white.

- Contrast Image Segmentation: Sometimes image contain unwanted data or some amount of noisiness data which is not provide good results at the time of testing. Hence to get the better results and to achieve something meaningful data form the image which helps to analyze the cancer easily we simplify image into multiple segments [14].

- Channel Standardization: In standardization it scales the pixels values at zero mean and unit variance. It supports two kinds of standardization i.e. either per image or per dataset [15].

\subsection{Augmentation Estimator}

This technique increase the performance of the estimator and gives the better results. In this method different techniques is used like cropping, rotating and flipping of the images [16] [12].

- Cropping: Extract only needful region from the overall region.

- Rotation: Rotate the image as per the user need and then perform the various operations to get the better results.

- Flipping: This part is used for reversing the image. In simple term it's a mirror image of original one.

\subsection{Segmentation}

Splitting operation performed on images in $2 \mathrm{X} 2,3 \times 3$ up to $10 \mathrm{X} 10$ patches we called it as segmentation [17]. In this segmentation process we train to the system to identify the close regions of interest which are important to detect the $\mathrm{BC}$. By eliminating unrelated data from the image it's easy to identify the tumor as early as possible. K-mean clustering algorithm is a method of groups it means similar objects combine in same group. Segmentation operation rely on it for better results and it gives better results when similar objects present in one group. It process fastly as compare scattered data [12]. Following figure 2 shows that how images is segmented and classify into different classes like 40x, 100x, 200x and 400x in two different attribute i.e. Benign (0) and Malignant (1) with their percentage ratio.

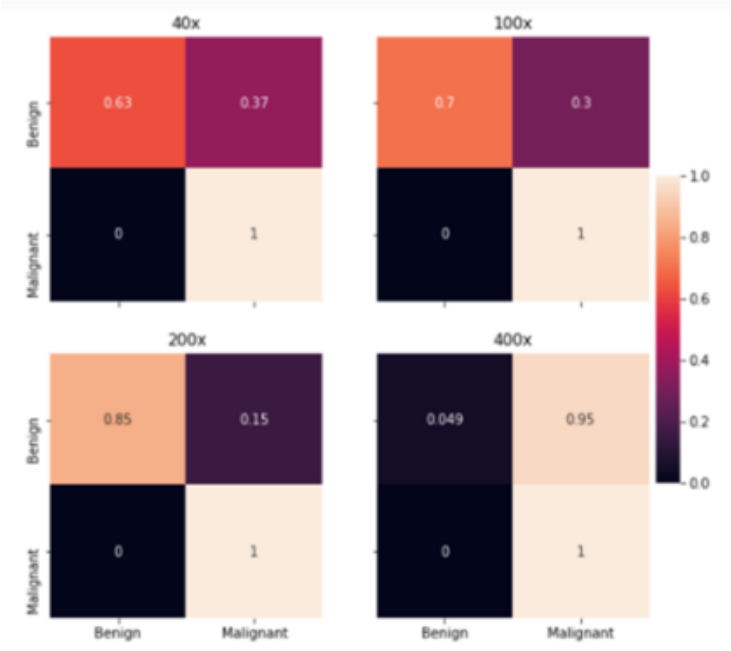

Figure 2 Segmented Data Evolution 


\subsection{Balancing}

This operation is important when we train our module manually. Sometimes we got the unbalanced data in percentage of Malignant and Benign in training phase. This unbalanced data effects on the performance or on the accuracy of the machine. If machine is heavily biased towards any type of tumor then its balancing is necessary to get the accurate results. So we use flipping technique to achieve the 50/50 proportion of Benign and Malignant.

- Thresholding:- Every image contain some part which is more useful than other part of same image and that part is very important to detect the $\mathrm{BC}$. The other less important part shown in lighter color without dark patches of cells. In this situation thresholding is used by setting up the value is 1 and identify the light pixel. After summing the value of patches the largest value used for classification [12].

\subsection{Preprocessing}

It increase the quality of the feature extraction method by removing unwanted data or pixel from image. It also apply some geometric transformations on images such as rotation, translation, scaling to highlight particular part of image.

Some image preprocessing methods are [18].

1. Local Binary Family Pre-Processing

2. Spectra Family Pre-Processing

3. Basis Space Family Pre-Processing.

Other train, model and outcome phases are used to training and testing purpose and at the end we got results in terms of accuracy of BC.

\section{METHODOLOGY}

With proper supervised learning to the machine in training phase which contain labels associated with the images helps to predict the label more accurately for new or an unseen images. Let's see the different Machine Learning techniques are used in this model with their outcomes. For training purpose and to process and check the accuracy of various algorithm, we are using a data set size is $18 \mathrm{~GB}$ which contain 5000 different images of BC.

\subsection{Convolutional Neural Network (CNN)}

Convolution is a signal processing operation which easily compute as a discrete spatial processing operation. Which is useful for 1D, 2D and 3D processing. The working behind this is, it combine two different signal together and change the source signal into filter one. This source signal is nothing but the array of pixel in the picture. This method is typically refer for some filtering operations like LowPass, HighPass and BandPass. But, most the filters detect the features from images via edge detection method or even with corner, point and contour detector. It is used in area are computer vision and Natural Language Processing [18].

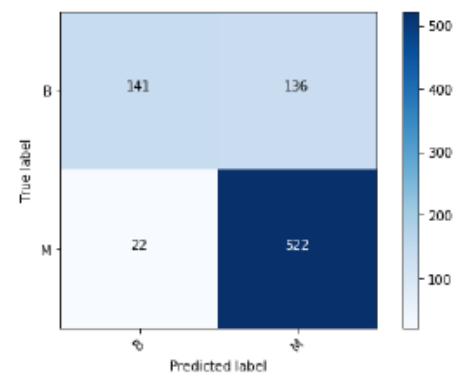

Figure 3 Confusion Matrix of CNN (Accuracy-79\%) 
Above figure shows that the Confusion Matrix of CNN. In this figure 3, 141 and 522 are the images which matches with the tumor and got the accuracy is $79 \%$.

\subsection{K-Nearest Neighbor (KNN)}

The algorithm used for the purpose of classification and regression is KNN which falls under category of supervised learning. The working of KNN is based on finding the points in the data which is close to the new point enter into the machine. Then algorithm sort it separately that closet points in terms of distance frame arrival point. This particular distance in point is measured by using different methodologies but Euclidian distance is mostly used by experts. In next phase, take a particular number of points whose distance is less as compare to other points and classify it into different category. Points are chosen in $\mathrm{KNN}$ is in the form of odd number, like number of classes is 2 similarly the highest number point is categories separately as a new data point [7].

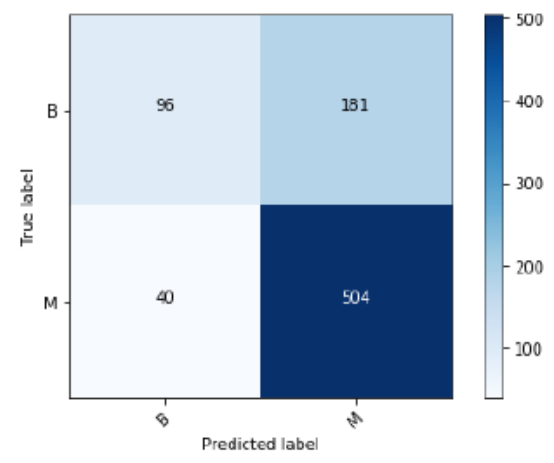

Figure 4 Confusion Matrix of KNN (Accuracy - 70\%)

Above figure shows that the Confusion Matrix of KNN. In this figure 4, 141 and 522 are the images which matches with the tumor and got the accuracy is $70 \%$.

\subsection{Inception $\mathrm{V} 3$}

Under Inception V3, we works on CNN called as InceptionNet. Which is the higher version of GoogleNet. CNN trained the machine with more than millions of images from the database. Inception V3 provide the platform where we can apply multiple filters for multiple inputs channel. The purpose of Inception V3 is, it reduce the high computational overhead if convolution layers by breaking the large convolution into a network of smaller convolution. Because of this method machine achieve the same degree of output in the less time for large convolutions. For example, the 1X1 convolution and Max-Padding apply on the large convolution to reduce the cost of 5 X5 convolutions. We can do it Inception V3 up to 48 Layer deep architecture but for our simplicity and need we work on 22 layer. We can increase of decrease the number of layers as per our requirements [12] [19].

\subsection{Support Vector Machine (SVM)}

As per the expert, the best technique for Machine Learning classification is SVM. We can use SVM with sorted data sets which is classify nearly into 2 classes. In multiple class classification, problem random partition of classes is chosen. Which is reduced it to the binary problem and perform it recursively [2]. Although SVM is a discriminative classifier works on separating Hyperplane [7]. It separates two classes by finding the best Hyperplane form all the points. To get the less generalization error then classifies the margin between the points must be larger and this can be achieved by the Hyperplane. That means the largest distance to the nearest 
training point of any class called good separation. The purpose of SVM is maximize the margin space between two classes of the Hyperplane (2).

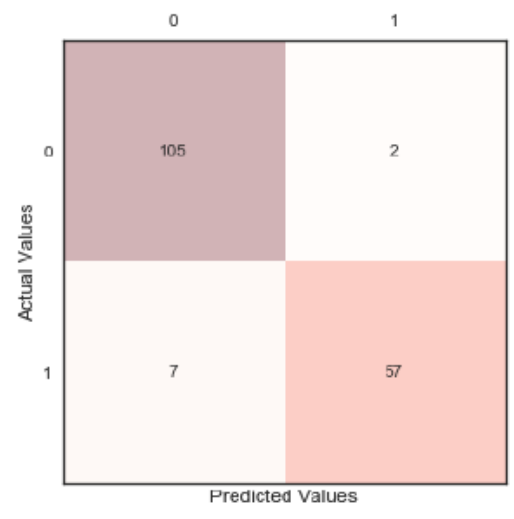

Figure 5 Confusion Matrix of SVM (Accuracy-95\%)

Above figure shows that the Confusion Matrix of SVM. In this figure 5, 105 and 57 are the images which matches with the tumor and got the accuracy is $95 \%$. The count of images in confusion matrix is increase or decrease on the basis of how much time we train the machine. Multiple times training increase the accuracy at the time of testing phase.

\subsection{Artificial Neural Network (ANN)}

It is a computational algorithm, which is based on the human neuron. Our brain contains millions of neurons. They sends the signal to each other and process it in the form of electrical and chemical signal. Synapses helps to neuron to passes the signal to other neurons. An ANN is nothing but the information processing technique and it works similar like human brain process the information. In ANN large number of units are connected together to process the information and gives the proper results. Not only for classification but also used for regression of continuous target attributes. ANN contain lots of power to process the data and in coming years ANN definitely grab the market [20].

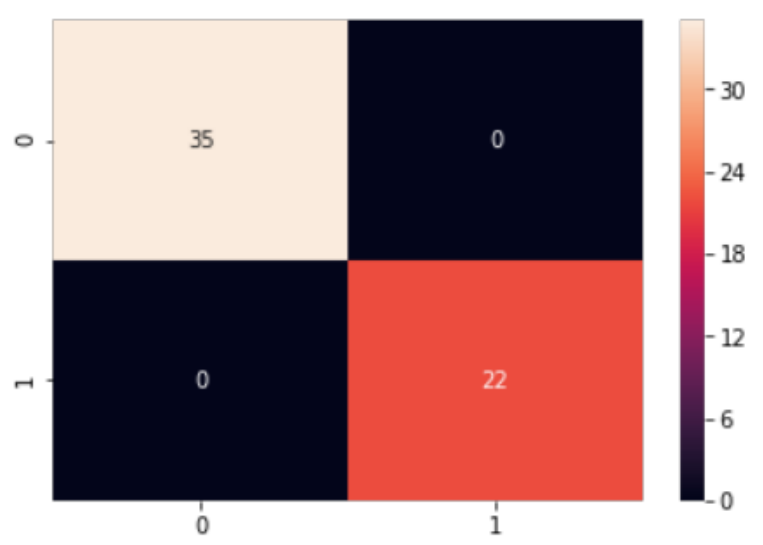

Figure 6 Confusion Matrix of ANN (Accuracy-99\%)

Above figure shows that the Confusion Matrix of ANN. In this figure 6, 35 and 22 are the images which matches with the tumor and got the accuracy is $99 \%$. At this point we train or machine so many times and output of SVM provide to the ANN and it gives better result. For better execution time we can take high configured machines or we can implement this module on cloud also. Implement cloud and combine the power of multiple systems and get the better results for processing the Dataset [21]. 
Let's analyze the results of SVM and ANN in training and testing phase and find the better method for breast cancer detection.

\section{EXPERIMENTAL RESULTS AND PERFORMANCE ANALYSIS}

In this model we applied different classification algorithms like CNN, KNN, Inception V3, SVM and ANN. The Confusion Matrix of all this technique as shown in methodology section. We performed this operation on the DataSet size is $18 \mathrm{~GB}$. This Data contain 5000 images which contain Benign and Malignant cells. This images help to identify the breast cancer by comparing normal images with this processed images and if the accuracy is high or find any tumor in breast then patient start taking prevention on this.

Let's first see the results of the module in training phase.

\begin{tabular}{|c|c|}
\hline \multicolumn{2}{|c|}{ Training Phase } \\
\hline SVM & ANN \\
\hline $95.10 \%$ & $99 \%$ \\
\hline $94.60 \%$ & $98.17 \%$ \\
\hline $95.40 \%$ & $98.52 \%$ \\
\hline $96 \%$ & $99.40 \%$ \\
\hline $95 \%$ & $99.30 \%$ \\
\hline
\end{tabular}

Figure 7 Training Phase Accuracy of SVM and ANN

Above table shows that the accuracy of SVM and ANN. Here, we provide input to the SVM is output of $\mathrm{CNN}, \mathrm{KNN}$ and inception V3. If we see that the accuracy provided by the CNN and $\mathrm{KNN}$ is $79 \%$ and $70 \%$ which is not so good for prediction of BC. Hence, we used another important technique i.e. SVM and we can see that the changes in accuracy after applying SVM. The accuracy is increased above $90 \%$ which is necessary in cancer detection. But, if see the results after applying ANN on SVM results it gives better accuracy. The more accuracy gives the correct results and the confirmation of which type of tumor is present or present or not in the cells. Let's the accuracy of SVM and ANN on graph.

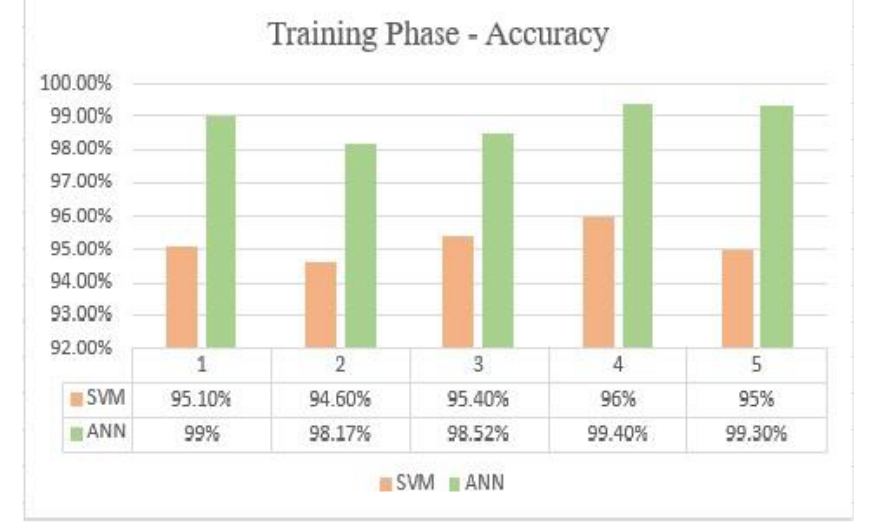

Figure 8 Training Phase Accuracy

Figure 8 shows that the accuracy of SVM and ANN. Results before and after applying ANN on SVM results. The graph clearly shown that the ANN gives far better results if we applied after the SVM stage. If the SVM alone gives accuracy up to the 98 or $99 \%$ then we not need to apply ANN but after applying ANN if you got good results then definitely go with the flow. The results of ANN depend the customization of layers and how much you train your module. Let's see the results in different graphical form. 


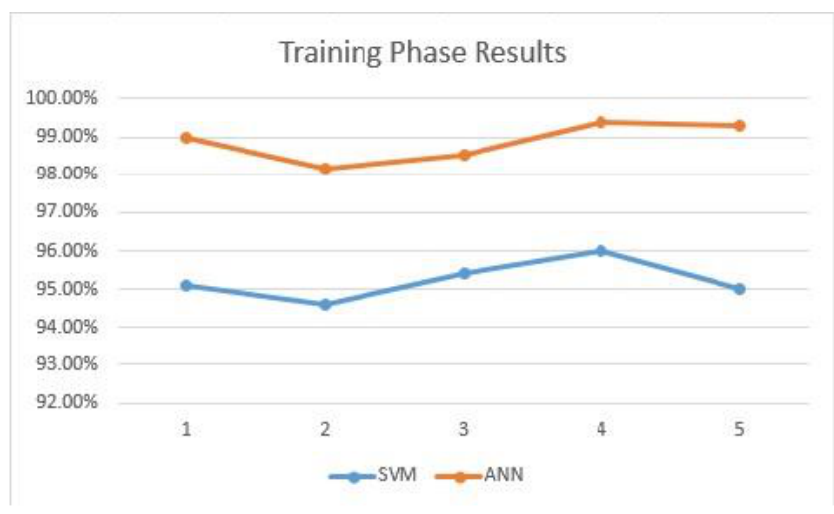

Figure 9 Training Phase Results

In figure 9 the orange line above the blue one represents the ANN results and the gap between the accuracy. In medical field this much amount of gap in accuracy is also causes many problems. It always better to make sure about your diseases by getting accurate results.

In similar manner, we train our model so many times and got the good results for ANN. Now we are going to test the actual results on the new images. Like a training, we provide new images to both the SVM and ANN model and analyze results.

\begin{tabular}{|c|c|}
\hline \multicolumn{2}{|c|}{ Testing Phase } \\
\hline SVM & ANN \\
\hline $90.00 \%$ & $97.42 \%$ \\
\hline $92.33 \%$ & $98.00 \%$ \\
\hline $92.40 \%$ & $95.62 \%$ \\
\hline $91 \%$ & $92.24 \%$ \\
\hline $90.15 \%$ & $99.00 \%$ \\
\hline
\end{tabular}

Figure 10 Testing Phase Accuracy of SVM and ANN

After providing new image to the SVM it provide accuracy in the range of 90 to $93 \%$ and the output of SVM give it to the ANN the accuracy is increase up to $99 \%$. The new images contains new data about cells hence its accuracy is less than training phase. Here we take 5 different images and the accuracy for that 5 images as shown in table. The following graph shows the performance of SVM and ANN.

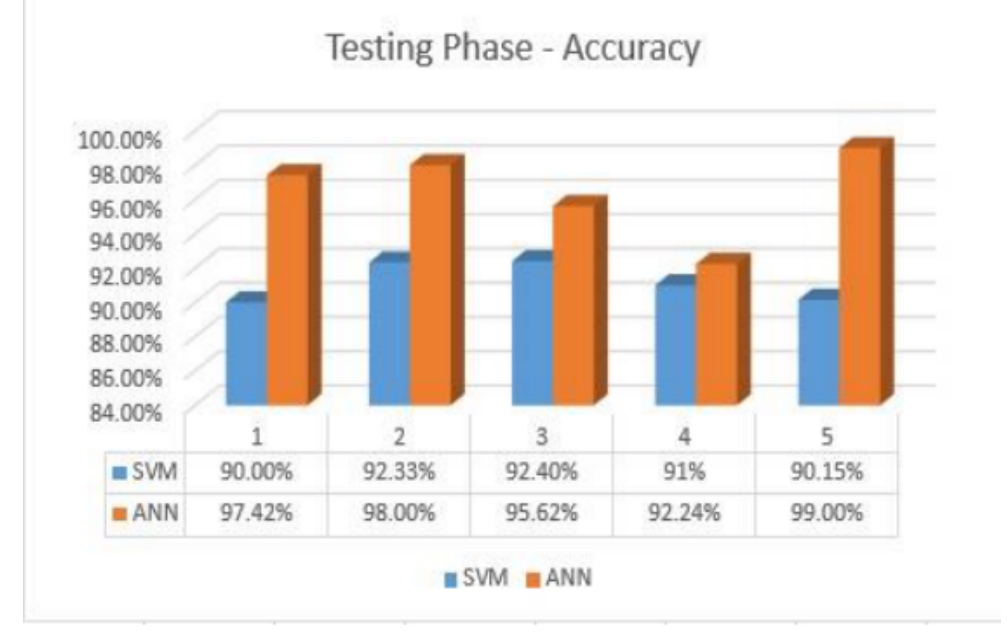

Figure 11 Testing Phase Accuracy 
In Testing phase also the accuracy of ANN after SVM in greater which is good for cancer detection. If we analyze the graph we can understand when the machine deals with completely new image and that kind of image is not train in training phase then it gives lower performance and if it gone through training the accuracy is good. The line graph shows the performance of SVM and ANN in the following.

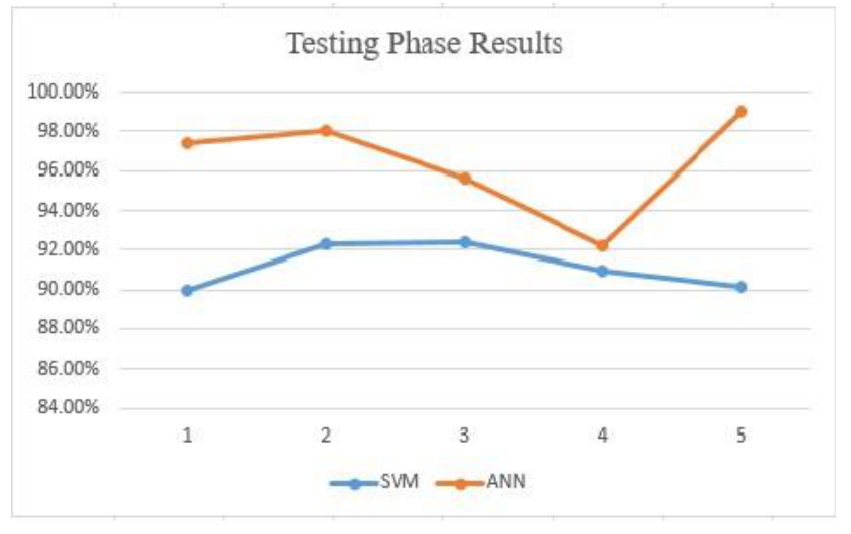

Figure 12 Testing Phase Results

In figure the orange line indicates the ANN results which is far better than SVM. We perform testing phase again and again we got the same results for this module. After analyzing the results of SVM and ANN in both the Training and Testing Phase we come on to the conclusion that, sometimes SVM alone gives the better accuracy without applying ANN on it. But, many of the times we got the good results after applying ANN on SVM results and graph of training and testing phase shows that how ANN gives good accuracy more than SVM.

\section{CONCLUSIONS}

In this paper we found out the best technique or algorithm for breast cancer detection with great accuracy. We saw how the cancer badly affects on the women and death ratio of it. We study the different techniques used before for the prediction of Breast Cancer in Literature Survey. Then we saw our proposed model for detection of BC and how its work. Then we go through the different algorithms used in our model i.e. CNN, KNN, Inception V3, SVM and ANN. We also saw the different Confusion Matrix of it after performing training phase. Then we analyze the results of the both the techniques SVM and ANN in Training and Testing Phase. The results says that, after applying ANN on SVM results we got accurate results with more accuracy. This results depends on the how we customize the ANN as per our requirements and how many times we train our data for getting good results. If we see the Testing Results the average accuracy of SVM we got is $91 \%$ and for ANN we got the $97 \%$ approximately. ANN definitely capture the medical field in coming year because it contains lots of power to process the data in more accurate form.

In future work we try to develop such kind of ANN model which gives good accuracy without SVM support. That means ANN alone can achieve the good accuracy. We also try to reduce the processing time of image by using better algorithms.

\section{ACKNOWLEDGMENTS}

We would like to thank Dr. P. B. Murnal. Dr. V. P. Kshirsagar, Dr. M. B. Nagori for their valuable support and guidance and Department of Computer Science and Engineering, GECA, Aurangabad for providing healthy environment for research work. 


\section{REFERENCES}

[1] B.M. Gayathri and Dr. C. P. Sumathi, "Comparative study of Relevance Vector Machine with various machine learning techniques used for detecting breast cancer", International Conference on Computational Intelligence and Computing Research, IEEE 2016.

[2] Dr. D. Selvathi and Aarthy Poornila A., "Performance Analysis of Various Classifiers on Deep Learning Network for Breast Cancer Dectection", International Conference on Signal Processing and Communication (ICSPC), pp. 359-363, IEEE 2017.

[3] Internet Sources, Link - https://www.mayoclinic.org/diseases-conditions/breast-cancer/symptomscauses/syc-20352470

[4] Internet Sources, Link - https://www.verywellhealth.com/what-does-malignant-and-benign-mean-14240

[5] Siyabend Turgut, Mustafa Dagtekin and Tolga Ensari, "Microarray Breast Cancer Data Classification Using Machine Learning Methods", 2018 Electric Electronics, Computer Science, Biomedical Engineerings' Meeting (EBBT), IEEE 2018.

[6] Phonethep Douangnoulack and Veera Boonjing, "Building Minimal Classification Rules for Breast Cancer Diagnosis", 2018 10th International Conference on Knowledge and Smart Technology (KST), pp. 278-281, IEEE 2018.

[7] Md. Milon Islam, Hasib Iqbal, Md. Rezwanul Haque and Md. Kamrul hasan, " Prediction of Breast Cancer Using Support Vector Machine and K-Nearest Neighbors", Region 10 Humanitarian Technology Conference( R10-HTC), pp. 226-229, IEEE 2017.

[8] S. Murugan, B. Muthu Kumar and S. Amudha, "Classification and Prediction of Breast Cancer using Linear Regression, Decision Tree and Random Forest", International Conference on Current Trends in Computer, Electrical, Electronics and Communication (ICCTCEEC), pp. 763-766, IEEE 2017.

[9] Ahmed Iqbal Priton, Shahed Anzarus Sabab, Md. Ahadur Rahman Munshi and Shihabuzzaman Shihab, " Predicting Breast Cancer Recurrence using effective Classification and Feature Selection technique", $19^{\text {th }}$ International Conference on Computer and Information Technology, pp. 310-314, IEEE 2016.

[10] Liu Lei, "Research on Regression Algorithm of Breast Cancer Diagnose Data by Machine Learning", International Conference on Robots \& Intelligent System, pp. 157-160, IEEE 2018.

[11] Lal Hussain, Wajid Aziz, Sharjil Saeed, Saima Rathore and Muhammad Rafique, "Automated Breast Cancer Detection using Machine Learning Techniques by Extracting Different Feature Extracting Strategies", $17^{\text {th }}$ International Conference on Trust, Security And Privacy In Computing And Communications/ $12^{\text {th }}$ International Conference On Big Data Science And Engineering, pp. 327-331, IEEE 2018.

[12] Internet Sources, Link https://drive.google.com/open?id=0Bz8g4zy1wCgFYk9FU2RMVINZSTcxdFEtSWFrN19PVEx5TIJB

[13] [13]. Deepika Verma and Dr. Nidhi Mishra, "Analysis and Prediction of Breast cancer and Diabetes disease datasets using Data mining classification Techniques", International Conference on Intelligent Sustainable System (ICISS), pp. 533-538, IEEE 2017.

[14] Zuiderveld, Karel. Graphics gems iv. In Heckbert, Paul S. (ed.), Graphics Gems IV, chapter Contrast Limited Adaptive Histogram Equalization, pp. 474-485. Academic Press Professional, Inc., San Diego, CA, USA, 1994.

[15] Internet Sources, Link- https://en.m.wikipedia.org/wiki/Image_segmentation

[16] Internet Sources, Link- https://machinelearningmastery.com/how-to-normalize-center-and-standardizeimages-with-the-imagedatagenerator-in-keras/

[17] Havaei, Mohammad, Davy, Axel, Warde-Farley, David, Biard, Antoine, Courville, Aaron, Bengio, Yoshua, Pal, Chris, Jodoin, Pierre-Marc, and Larochelle, Hugo. Brain tumor segmentation with deep neural networks. Medical image analysis, 35:18-31, 2017. Internet
https://pdfs.semanticscholar.org/cc43/a71e05cfc49ab0777b82ca94d181f779149f.pdf

Link- 
[19] Internet Sources, Link- https://www.mathworks.com/help/deeplearning/ref/inceptionv3.html

[20] Internet Sources, Link- https://www.datasciencecentral.com/profiles/blogs/artificial-neural-network-annin-machine-learning

[21] Nikhil Wagh, Vikul Pawar and Kailash Kharat, "Implementation of Stable Private Cloud using OpenStack with Virtual Machine Results", International Journal of Computer Engineering \& Technology (IJCET-19), Volume 10, Issue 2, and March-April 2019.

Research Scholar Kalyani R. Wadkar: Received the B.E.degree in Computer Science and Engineering fromProf.Ram Meghe Institute of Technology \& Research, Amravati under Sant Gadge Baba Amravati University, Amravati, Maharashtra, India, in 2014, and currently persuing M.E degree in Computer Science and Engineering from Government College of Engineering, Aurangabad under Dr. BabasahebAmbedkar Marathwada University, Aurangabad, Maharashtra, India. I have 2 years ofExperience as Database Administrator in Tech Mahindra LTD Pune India during 2014-2016.

Prof. Prashant D. Pathak: Received the B.E.degree in Information Technology from Government College of Engineering Aurangabad, under Dr. Babasaheb Ambedkar Marathwada University, Aurangabad, Maharashtra, India, in 2007. and the M.E degree in Computer Science and Engineering from Government College of Engineering, Aurangabad under Dr. Babasaheb Ambedkar Marathwada University, Aurangabad, Maharashtra, India, in 2013. He has 7 years of professional experience. He worked as web developer and software developer and have a 2 year of experience. Currently his working as Assistant Professor in Government College of Engineering, Aurangabad.

Research Scholar Nikhil R. Wagh: I have completed B.E. in Computer Science and Engineering in 2017 and currently doing M.E. from Government College of Engineering Aurangabad. I have published two paper first paper is - "Educational Cloud Framework - A Literature Review on Finding Better Private Cloud Framework For Educational Hub", International Conference on Emerging Technologies in Computer Engineering: Micro services in Big Data Analytics (ICETCE), Jaipur, India, Springer 2019.

Second paper is- "Implementation of Stable Private Cloud using OpenStack with Virtual Machine Results", International Journal of Computer Engineering \& Technology (IJCET-19), Volume 10, Issue 2, and March-April 2019. Interested research area is Cloud Computing and I am working on it from past 2 years. 